\title{
MEASURING CONSUMER-BASED BRAND AUTHENTICITY
}

\author{
Julie Napoli, Curtin University, Australia \\ Sonia J. Dickinson, Curtin University, Australia \\ Michael B. Beverland, University of Bath, United Kingdom \\ Francis Farrelly, Royal Melbourne Institute of Technology, Australia
}

\begin{abstract}
Authenticity has historically been associated with transcending the self and the market (Beverland, 2005; Fine, 2003; Kozinets, 2002; Peterson, 2005; Thompson, Rindfleisch, \& Arsel, 2006), yet an emerging stream of consumer research identifies that people attribute authenticity to brands. Research suggests that authenticity is central to brand status, equity and corporate reputation (Beverland, 2005; Gilmore \& Pine, 2007), with some even suggesting it is one of the "cornerstones of contemporary marketing", (Brown, Kozinets \& Sherry, 2003, p. 21). Scholars have previously examined consumers' quests for authentic experiences (Arnould \& Price, 2000), rituals associated with the authentic self (Belk \& Costa, 1998; Kozinets, 2002), the cues used to attribute authenticity to objects (Beverland, Lindgreen, \& Vink, 2008; Grayson \& Martinec, 2004; Leigh et al., 2006; Thompson et al., 2006), the processes used to assess an object's authenticity (Rose \& Wood, 2005), and the various forms that authenticity can take (Brown et al., 2003; Grayson \& Martinec, 2004). We extend this line of inquiry by defining and measuring consumer-based brand authenticity. We do this through the development of a brand authenticity scale. Such research seeks to reduce the present fragmentation of research on the consumption of authenticity and assist managers create and maintain a brand's authenticity_-building on calls for more research in this area (Beverland, 2005; Leigh et al., 2006; Peterson, 2005).
\end{abstract}

Four separate studies were undertaken to generate and refine scale items (Study 1), determine and then confirm the underlying factor structure of brand authenticity (Studies 2 and 3), and finally to test for convergent and discriminant validity of the scale (Study 4). In Study 1, Churchill's (1979) scale development paradigm was adopted, using a deductive approach for the generation of scale items (Schwab, 1980). Drawing on the extant literature, an initial list of 157 items was generated reflecting brand heritage (26 items), quality commitment (21 items), craftsmanship (14 items), sincerity (43 items), nostalgia (23 items), cultural symbolism (21 items) and design consistency (nine items). Content validity of the initial pool of items was then assessed following the method outlined by Bearden, Netemeyer and Teel (1989) and Zaichkowsky, (1985), resulting in the retention of 33 scale items. Study 2 involved further purification of the scale and an assessment of the internal reliability of the 33 items. Data was collected via self-administered questionnaires from 247 undergraduate students at a large inner city university. Several items were eliminated during analysis, resulting in the retention of 19 items reflecting three factors, namely quality commitment, heritage and sincerity. These items formed the basis for further structural testing through confirmatory factor analysis in study 3, where data was gathered from a second sample of university students $(\mathrm{n}=203)$. Examination of the data suggested the deletion of five additional items, resulting in 14 items representing three factors as follows: quality commitment (seven items, $\alpha=.879$ ), sincerity (two items, $\alpha=.605$ ) and heritage (five items, $\alpha$ $=.783)$. The fit statistics for the three factor model were chi-square $=100.84,74$ degrees of freedom $(\mathrm{p}=.021)$; comparative fit index $(\mathrm{CFI})=.976$, Non-normed fit index $(\mathrm{TLI})=.971$, Normed Fit Index $(\mathrm{NFI})=.917$, and the root mean square error of approximation $(\mathrm{RMSEA})=.042$, which are within the guidelines recommended by Kelloway (1998). The estimates for the composite reliability and average variance extracted for each construct also attest to the internal consistency of the scale. Study 4 was designed to evaluate the construct validity of the BA scale in relation to measures of brand trust and brand credibility. Data was collected from 312 adult consumers, with results demonstrating both convergent and discriminant validity of the BA scale.

In this study we broaden our understanding of brand authenticity as a multidimensional construct by developing a psychometrically robust measure of brand authenticity. By being able to measure and assess authenticity, marketers may be empowered to identify new opportunities for brand positioning and value creation that may contribute to greater consumer loyalty and attachment to a brand. Moreover, a robust measure of brand authenticity enables managers to track performance over time, which can help direct future advertising strategies and decisions on brand extensions, sponsorship choices and cobranding partnerships. Future research could be directed toward determining variations in authenticity evaluations across levels of involvement, level of branding and types of goods. Authenticity evaluations may also vary across cultures so it would be relevant to examine the features that are shared amongst among different cultural groups as this has implications for managing brands across cultures.

References available upon request 